\title{
Specific signal imaging velocimetry for rarefied plasma flows
}

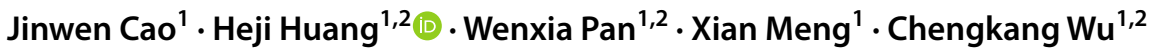 \\ Received: 23 January 2020 / Revised: 14 October 2020 / Accepted: 16 October 2020 / Published online: 8 November 2020 \\ (c) Springer-Verlag GmbH Germany, part of Springer Nature 2020
}

\begin{abstract}
High-velocity and rarefied plasma flow has found great application in aerospace science and technology. However, it is difficult to characterize such a flow using traditional methods based on the continuous medium flow hypothesis. With principles similar to those of particle imaging velocimetry, a specific signal imaging velocimetry method is proposed to measure the fluid velocity. Instead of using tracing particles, a specific signal was used as a fingerprint, which facilitated better fluid followability and measurement accuracy. As a verification example, the axial velocity distribution of a plasma plume along the flow direction was experimentally measured. The results agreed well with intrusive electrostatic probe measured values, which shows that SSIV has a great potential for fast and two-dimensional velocity measurements in similar flows. Considering the abilities and requirements of the experimental equipment and the signal processing techniques, the applicability of the proposed specific signal imaging velocimetry method was discussed.
\end{abstract}

Electronic supplementary material The online version of this article (https://doi.org/10.1007/s00348-020-03080-9) contains supplementary material, which is available to authorized users.

Extended author information available on the last page of the article 


\section{Graphic abstract}

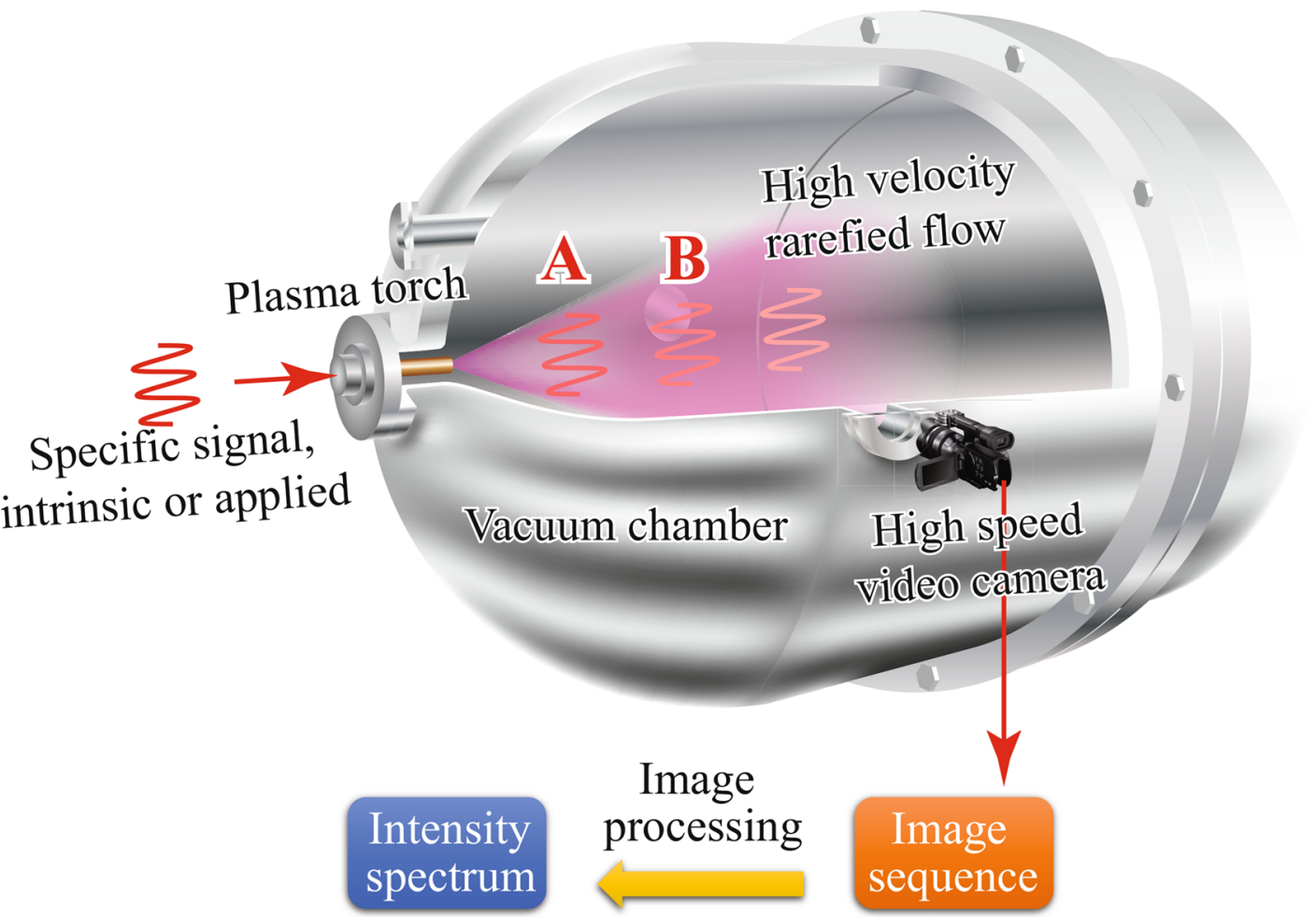

Signal

Analysis
Time
shift
Statistics
Velocity
field

Instead of using tracing particles, a specific signal, intrinsic or applied was used for imaging velocimetry with better followability and accuracy.

\section{Primary symbols}

A Amplitude

D Diameter

$f \quad$ Frequency

I Intensity

$L \quad$ Characteristic length

$m \quad$ Integer number

$n \quad$ Integer number

$N \quad$ Integer number

px Pixel

$r \quad$ Radial position

$t \quad$ Time

$U \quad$ Velocity of fluid flow

$v \quad$ Environmental velocity

$w \quad$ Width $x \quad$ Axial position

$\varepsilon \quad$ Dissipation rate per unit mass

$\mu \quad$ Viscosity

$\rho \quad$ Density

$\tau \quad$ Characteristic time

$v \quad$ Kinematic viscosity

$\varphi \quad$ Phase of a signal

\section{Abbreviations}

CCD Charge-coupled device

DC Direct current

FFT Fast Fourier transformation

FOV Field of view

MTV Molecular tagging velocimetry

OSR Object space resolution

PIV Particle imaging velocimetry 


\begin{tabular}{ll} 
RF & Radio frequency \\
SSIV & Specific signal imaging velocimetry \\
WD & Working distance \\
\multicolumn{2}{c}{ Notations } \\
0 & Initial value \\
$\infty$ & Infinite value \\
$a$ & Camera \\
$i$ & Index number in axial direction \\
$j$ & Index number in radial direction \\
max & Maximum value \\
$\min$ & Minimum value \\
$p$ & Particle \\
$p x$ & Pixel \\
$s$ & Specific signal
\end{tabular}

\section{Introduction}

The conversion and use of energy is closely related to human life. In a sense, it can even be said that most production and life processes are energy conversion and utilization processes. Industrial applications of thermal plasma are no exception. For more than a century, thermal plasma has played an important role in a wide variety of applications, such as material spraying and coating (Fauchais and Vardelle 1997; Kambara et al. 2014; Pfender 1999), surface modification, gas heating for wind tunnels (Chazot and Panerai 2015; Dubreus et al. 2013), plasma propulsion (Johnson et al. 2015; Pan et al. 2016; Wilson 2006), and so on. Although these applications are diverse, from the perspective of energy utilization, the main focus is on converting electrical energy into plasma internal energy and finally converting it into the preferred thermal, chemical, and kinetic energy and using it. Most thermal plasma applications use multiple forms of energy at the same time, although one form may dominate. For example, plasma cutting/welding primarily uses thermal energy, as does thermal plasma spraying. The high temperature and high enthalpy of thermal plasma make the cutting/welding of thick high melting point metal plates possible (Metcalfe and Quigley 1975; Ramakrishnan et al. 1997) and enable refractory metals and high melting point ceramics to be sprayed to form the required coatings (Fauchais et al. 2014). There are also applications that use a single energy form. For example, in an arc-heated electrothermal space thruster, one of the most important efforts is to convert as much of the input energy as possible into the kinetic energy of the propellant to improve the thrust efficiency and the specific impulse (Ahedo 2011; Auweter-Kurtz et al. 1996; Mazouffre 2016). Another example is using a high enthalpy plasma flow as the source for a hypersonic rarefied wind tunnel to simulate vehicle flight conditions, where the incoming flow must fulfill the similarity criterion of binary scaling law (Shen 2005), namely, two flows are similar when they have the same absolute velocity $\left(v_{\infty}\right)$ and the same product of the flow density and characteristic length $\left(\rho_{\infty} L\right)$. In these applications, where kinetic energy is of more concern, accurate measurement of fluid velocity is of particular importance.

Based on different measurement principles, fluid velocity measurement methods can be divided into three categories. The first category is energy-based, including pressure-based velocity measurements (Brossa and Pfender 1988), thermal anemometry (Lekakis et al. 1989), etc., in which the corresponding fluid velocity is obtained through energy inversion; the second category is Doppler effectbased, among which the laser Doppler anemometer is a representative one, measuring the Doppler shift to determine the velocity of fluid markers (Farmer and Brayton 1971; Fauchais et al. 1989; Lyn et al. 1995); the third category is speed definition-based, in which the velocity of the target is obtained by measuring the displacement of the target within a certain time. Molecular tagging velocimetry (Gendrich et al. 1997; Lempert et al. 2002; Segall et al. 1996; Wehrmeyer et al. 1999), particle image velocimetry (Keane and Adrian 1990; Raffel et al. 2017; Westerweel et al. 2013) and so on belong to this category. For compressible flow characterizations, the Schlieren visualization method is also widely applied (Underwood et al. 2020), and velocity measurement can also be conducted by cinematic Schlieren diagnostics (Wang et al. 2019).

However, conventional velocity measurement methods and equipment are not suitable for certain occasions. Among them, the previously mentioned high-velocity rarefied plasma flow is an example. On one hand, such a flow usually deviates from thermodynamic equilibrium, which hinders the application of energy-based measurements, such as the Pitot tube (Brossa and Pfender 1988; Fauchais et al. 1989) and cross-wire anemometry (Acrivlellis 1980; Kawall et al. 2006; Lekakis et al. 1989); on the other hand, the applicability of particle-based methods is also limited because the accuracy of the measurement largely depends on the followability of the tracing particles, which can be very poor in rarefied flow conditions. For example, the response time of the particle tracking the fluid is (Raffel et al. 2017)

$\tau_{\mathrm{P}}=\frac{d_{\mathrm{P}}^{2}}{3} \frac{\rho_{\mathrm{P}}}{18 \mu}$.

The fluid viscosity $\mu$ decreases to zero with pressure (Chapman and Cowling 1958). Hence, the response time of the tracing particles is quite large in a low-pressure flow. When dealing with a plasma plume under reduced pressure, the rarefied feature is much more significant because of the high temperature and low pressure of the plume. As studied 
in a thermal plasma jet with a velocity of $2500 \mathrm{~m} / \mathrm{s}$ under a pressure of $2 \mathrm{kPa}$, the seeded particles with a diameter of $\sim 5 \mu \mathrm{m}$ obtained a velocity of only $500 \mathrm{~m} / \mathrm{s}$ (Fauchais 2004; V et al. 2003), which is comparable with the typically used tracing particles in PIV (Kohli 2012). Furthermore, oil droplets, which are normally used as PIV tracing particles, cannot survive long enough in thermal plasma because of the high temperature and high chemical reactivity.

Efforts have been made to properly measure the velocity of rarefied plasma flows. For instance, electrostatic probes were used to measure plasma-flow velocity based on several methods (Bufton and Burton 1997; Johnson and Murphree 1969; Kado et al. 2004; Maclatchy et al. 1992; Peterson et al. 1994). However, this method is intrusive. An effective way to avoid intrusion is to use the optical emissions of the plasma. For example, Coudert, etc. (Coudert et al. 1995; Planche et al. 1998) have measured the axial velocity of the plasma jet exiting a direct current (DC) plasma torch by recording the transmitting of the optical fluctuation, taking advantage of the restrike phenomenon of arc plasma that causes the fluctuation. However, these two methods could barely obtain the axial velocity distribution within a short time interval because a point-by-point measuring procedure is needed, while instantaneous measurement of the axial velocity distribution is useful, especially when evaluating the influence of the flow unsteadiness on the concerned parameters. Molecular tagging velocimetry (MTV) can instantaneously obtain the velocity distribution by continuously tracking the fluorescence of laser tagged molecules (Gendrich and Koochesfahani 1996; Gendrich et al. 1997; Lempert et al. 2002; Segall et al. 1996; Wehrmeyer et al. 1999) with a fast CCD camera. The laser beam must be chosen properly to excite or dissociate molecules, such as $\mathrm{H}_{2} \mathrm{O}$ and ozone. Recently, an MTV based on NH fluorescence was developed for velocity measurement of hypersonic rarefied gas flow (Zhang et al. 2017). However, the features of short-lived fluorescence (Lempert et al. 2002) and the dependence of fluorescence on molecular species remain issues.

To counter these difficulties, specific signal imaging velocimetry (SSIV) was proposed in this paper. Instead of using particles, a specific signal, either intrinsic or applied, was used as the tracer. To some extent, this method can be considered a type of optical flow method (Optical Flow and Trajectory Estimation Methods 2016). The detailed methodology is explained in the next section. To verify the SSIV principle, experimental measurements of the axial velocity distributions in a direct current argon plasma plume in a laminar flow state were first conducted using an intrinsic modulation of the plasma input energy and the corresponding optical emission signal of the plume as the tracing signal. Additional experiments for turbulent flow were also conducted where an externally applied specific signal was used. (a)

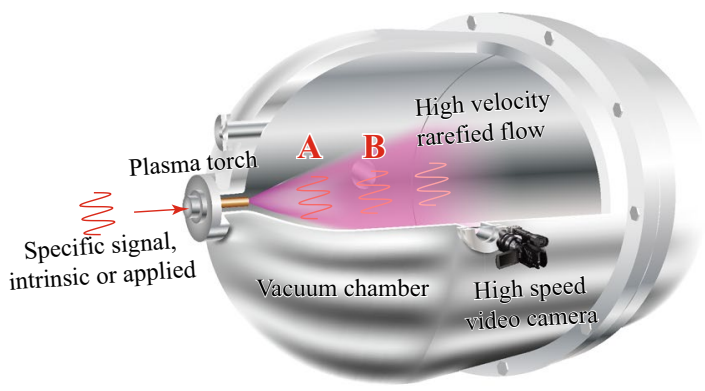

(b)

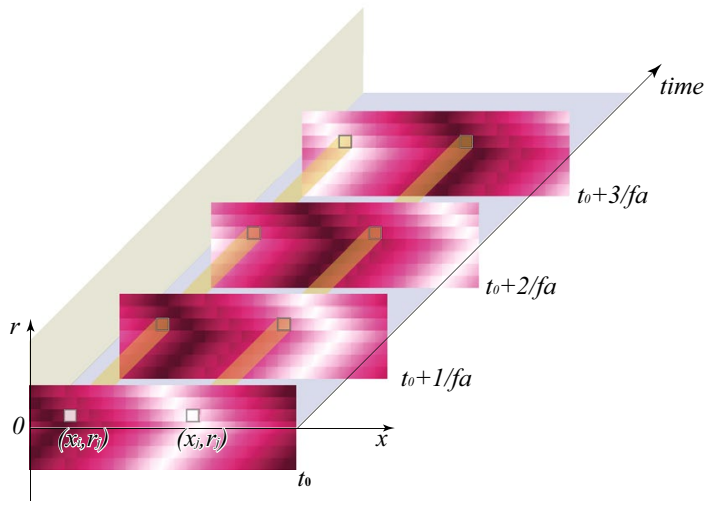

(c)

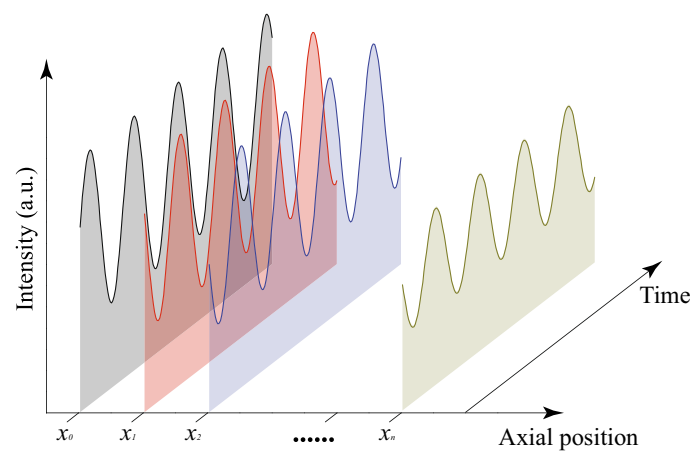

Fig. 1 Illustration of the specific signal image velocimetry for plasma flows. a Is the experiment setup of SSIV; $\mathbf{b}$ illustrates image frames obtained using a high-speed video camera; and $\mathbf{c}$ illustrates possible special and temporal variations of the specific signal at points with different axial positions if the specific signal is in a sinusoidal form

\section{Methodology}

Comprehensive consideration of the tracers used in particlebased velocimetry has been discussed in a wealth of literature (Raffel et al. 2017). As a tracer, an ideal specific signal should also have a slight interference with the flow field to be measured and be easy to distinguish. In SSIV, the specific signal is based on a series of small volumes with different states of the flow in the time domain. It is the pattern of such a signal, normally the amplitude and frequency that makes it distinguishable and trackable. Notably, as long as the signal can be identified from the background by some 
method, whether the pattern becomes fuzzy is not important. From this perspective, an intrinsic electromagnetic signal, such as the fluctuation of the plasma energy (Huang et al. 2010) at a specific frequency, can be a good tracer candidate for a high-speed rarefied plasma plume. Externally applied electromagnetic signals can also play the same role if the basic requirements for tracers are met.

Usually, the plasma is produced by direct current (DC) or radio frequency (RF) discharges (Boulos et al. 1997; Zhukov and Zasypkin 2007), or even by the hybrid type (Huang et al. 2003), where the electrical energy is applied to the feeding gas in an elaborately designed torch. Considering a small volume of plasma passing through the field where the electrical energy is applied, if its residence time inside such a field is smaller than the period of the input energy variation, a "stamp" of the plasma state, at either a high or low energy state, will not be averaged and will follow with the movement of the small volume of the plasma. The combination of a series of such small volumes with different states of the flow in the time domain makes the specific signal required for SSIV.

Therefore, when a periodic change of the plasma input energy occurs, a corresponding time-dependent variation of the plasma optical emission intensity $I(t)$ can be observed, as illustrated in Fig. 1. As mentioned above, although the absolute intensity of the optical emission might change in the flow field for possible decay of plasma density and temperature, the fluctuation behavior remains detectable, and the "stamp" is recognizable. By tracking the movement of the "stamp", the velocity distribution is obtained.

A simple way to capture the "stamp" is to use a highspeed video camera to record the plasma optical emission intensity, resulting in a frame sequence of $2 \mathrm{D}$ spatial

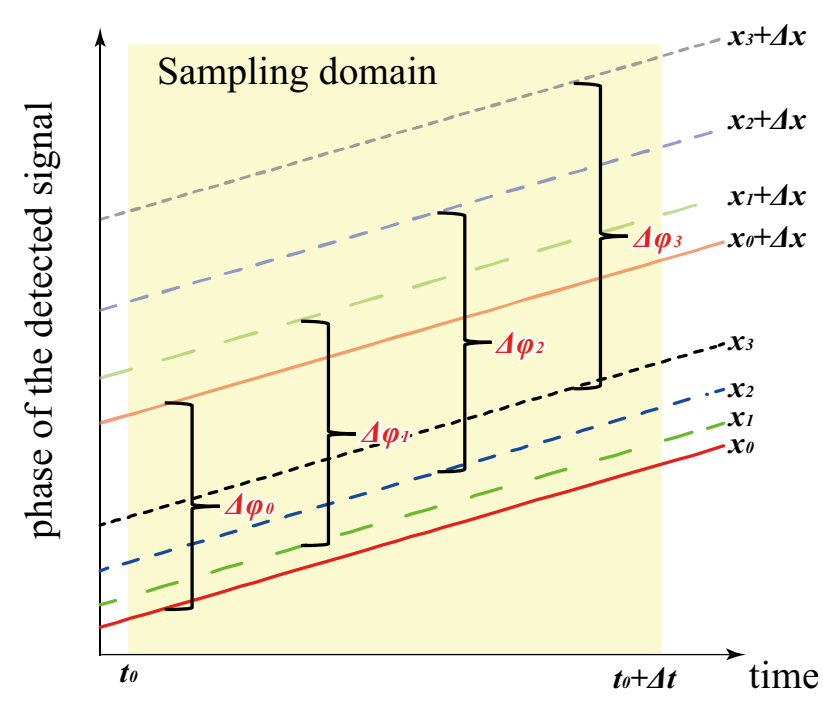

Fig. 2 Schematic diagram of the phase shift analysis within a sampling domain intensity with a temporal interval of $1 / f_{a}$ and a total number of $N$, as shown in Fig. 1b. Each pixel of the image corresponds to a small area on the focal plane. The correspondence between the image and the physical geometry is obtained by prior calibrations. For each small area in the focal plane, a time-dependent variation of the intensity occurs as a result of the intrinsic or applied specific signal. The temporal fluctuations of all spatial positions were obtained by extracting the optical intensity at the same position in every frame of a sequence, for example, position $\left(x_{i}\right.$, $\left.r_{j}\right)$ and position $\left(x_{j}, r_{j}\right)$ shown in Fig. 1b. Figure 1c illustrates possible special and temporal variations of the specific signal at points with different axial positions if the specific signal is in a sinusoidal form. Because the distance between two points is already known, a simple and direct way to calculate the mean velocity between them is by extracting the propagating time difference of the respective curves by tagging the crests or the troughs of the fluctuations. However, there can be large errors in tagging the crests or the troughs, and the error increases if the spatial interval is too small. Using a phase shift in the frequency domain is beneficial for a more precise determination of the propagation time. It should be noted that static or quasistatic flow within the propagating time is assumed; otherwise, the calculated velocity will not show the transient value correctly. This assumption will be met if the propagating time considered is sufficiently small compared with the characteristic time of the flow state, which will be discussed later.

For simplicity, the axial velocity distribution in a laminar plasma flow with a specific sinusoidal signal having one dominant frequency $f_{\mathrm{s}}$ is first considered.

The detected intensity of the signal for each position can be expressed as:

$I\left(x_{i}, t\right)=A\left(x_{i}\right) \sin \left[2 \pi f_{\mathrm{s}}\left(t-\int_{0}^{x_{i}} \frac{d x}{U(x)}\right)\right]$,

where $A\left(x_{i}\right)$ is the specific signal amplitude at position $x_{i}$.

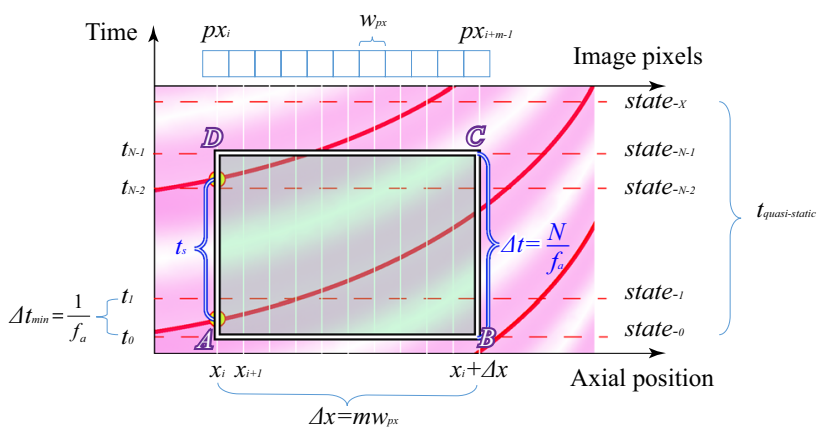

Fig. 3 Considerations for optimizing SSIV experimental parameters. The light and dark stripes illustrate possible temporal and spatial distributions of the flow intensity, with solid lines indicating the periods of variation 
Because the high-speed camera images are shot from the side perpendicular to the flow direction, the frequency shift due to the Doppler effect is negligible. Therefore, $f_{\mathrm{s}}$ can be considered a constant parameter. Thus, the phase increase is linear with time and has the same rate at every position, as demonstrated in Fig. 2, which means that although the absolute phase at each position varies with time, the phase shift between two positions remains unchanged at the same moment. An increase in $\varphi$ and $\Delta \varphi$ with axial position is also illustrated in Fig. 2, which indicates a decrease in velocity along the flow direction. In a sampling time of $\Delta t$, which is an integer multiple of the shortest temporal resolution of $1 / f_{\mathrm{a}}$, there might be several periods of the signal, and the initial phases at each position are used for calculation. The measured velocity is the mean value between two points with a spatial step of $\Delta x$, which is also an integer multiple of the highest spatial resolution determined by the specifications of the imaging system, including the CCD resolution, lens FOV and the distance between the target flow and the camera.

At an arbitrary moment $t_{0}$, the dependence of the phase shift between positions $x_{i}$ and $x_{i}+\Delta x$ on the velocity distribution can be calculated as:

$\Delta \phi_{i}=\phi\left(t_{0}, x_{i}\right)-\phi\left(t_{0}, x_{i}+\Delta x\right)=2 \pi f_{\mathrm{s}} \int_{x_{i}}^{x_{i}+\Delta x} \frac{d x}{U(x)}$.

Based on the mean value theorem, the mean velocity between two points can be calculated from the following equation:

$\bar{U}\left(x_{i}\right)=\frac{\Delta x}{\int_{x_{i}}^{x_{i}+\Delta x} \frac{d x}{U(x)}}=2 \pi f_{\mathrm{s}} \frac{\Delta x}{\Delta \phi_{i}}$.

Because $\varphi\left(t_{0}, x_{i}\right)$ and $\varphi\left(t_{0}, x_{i}+\Delta x\right)$ can be obtained through experimental measurement and signal inversion, $\bar{U}\left(x_{i}\right)$ can be calculated. By continuously repeating these processing procedures, SSIV can diagnose the dynamic flow field with a temporal resolution of $\Delta t$ and spatial resolution of $\Delta x$.

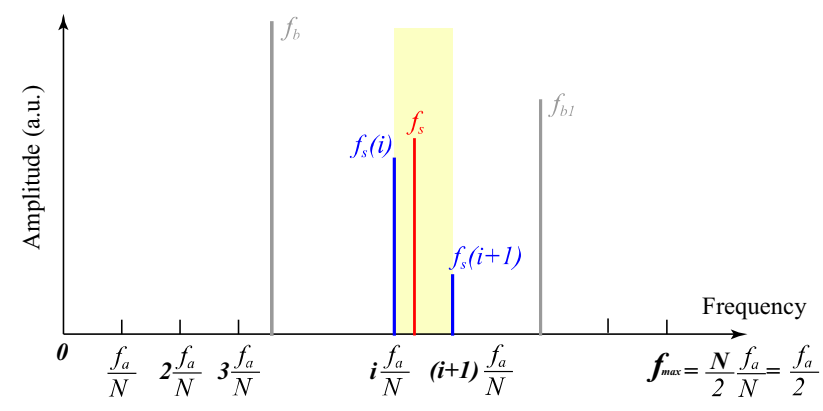

Fig. 4 Distinguishable range and accuracy of frequencies using FFT
Experimental parameters should be carefully selected to obtain appropriate $\Delta t$ and $\Delta x$ ranges to balance the measurement accuracy and precision. For the ABCD domain used for velocity calculation with a time-space geometry of $\Delta t \times \Delta x$ shown in Fig. 3, some restrictions should be met:

(1) According to the Nyquist-Shannon sampling theorem, the sampling rate should exceed the objective signal's frequency by at least a factor of two. In SSIV, the sampling rate is the frame rate of the high-speed video camera $\left(f_{\mathrm{a}}\right)$, and the objective signal's frequency is the specific frequency of $f_{\mathrm{S}}$. These settings mean that $f_{\mathrm{a}}>2 f_{\mathrm{s}}$ should be met.

(2) $\Delta t$ should be larger than at least one period of the specific signal; otherwise, complete acquisition of the specific signal is not possible, which gives the restriction $\frac{N}{f_{\mathrm{a}}}>\frac{1}{f_{\mathrm{s}}}$

(3) $\Delta t$ should be smaller than a certain time $t_{\text {quasi-static }}$, which means that during this time period, the flow state does not change substantially and can be considered quasistatic;

(4) $\Delta x$ can be as small as the object space resolution of the imaging system, which is closely related to the characteristics of the video camera, including the image sensor size, the sensor resolution, the angular FOV of the lens and the working distance between the camera and the objective flow. For noise reduction, $\Delta x$ should be greater than the object space resolution, which gives $\Delta x>\frac{\tan \left(\frac{\text { FOV }}{2}\right) \times W D}{\text { Sensor_Resolution }}$. For example, when using a camera with a CCD resolution of 512 in the $x$ direction, a lens with an angular FOV of $47^{\circ}$, and the object is at a distance of $300 \mathrm{~mm}, \Delta x$ should be greater than $\frac{\tan \left(\frac{47^{\circ}}{2}\right) \times 300}{512}=0.254 \mathrm{~mm}$.

(5) $\Delta x$ should not be too large, because SSIV measures the mean velocity through $\Delta x$ within the sampling time $\Delta t$, and $\Delta x$ determines how close the measured value is to the local velocity.

Fast Fourier transformation (FFT) is effective in detecting a specific signal in the frequency domain. As a discrete method, the distinguishable range and accuracy of frequencies are largely dependent on the sampling rate and the total number of samples, which corresponds to $f_{\mathrm{a}}$ and $N$ in SSIV, as shown in Fig. 4. The maximum frequency equals $\frac{f_{\mathrm{a}}}{2}$, with intervals of $\frac{f_{\mathrm{a}}}{N}$. To identify $f_{\mathrm{s}}$, where $i \frac{f_{\mathrm{a}}}{N}<f_{\mathrm{s}}<(i+1) \frac{f_{\mathrm{a}}^{2}}{N}$, background signals $\left(f_{\mathrm{b}}, f_{\mathrm{b} 1}\right.$, etc.) should be sufficiently far from this range. Because the frequency $f_{\mathrm{s}}$ might not be exactly distinguished at limited $f_{\mathrm{a}}$ and $N, f_{\mathrm{s}}(i)$ or $f_{\mathrm{s}}(i+1)$ is actually used for a velocity calculation. From this perspective, a small deviation from $f_{\mathrm{s}}$ owing to a frequency shift will not 


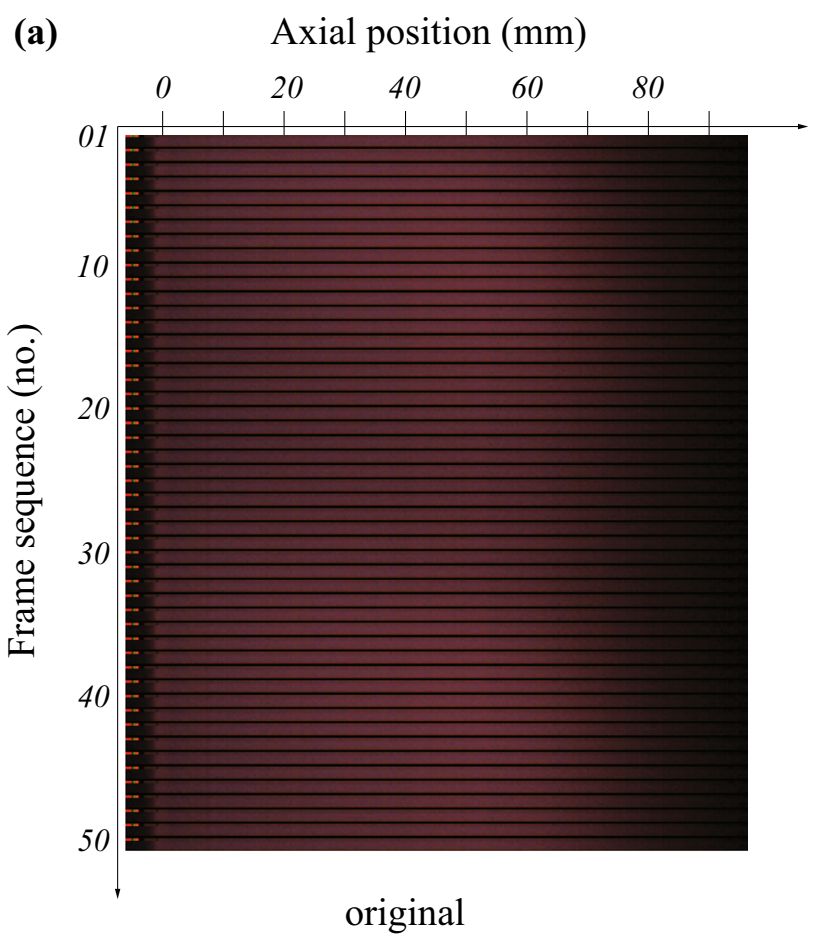

(a)

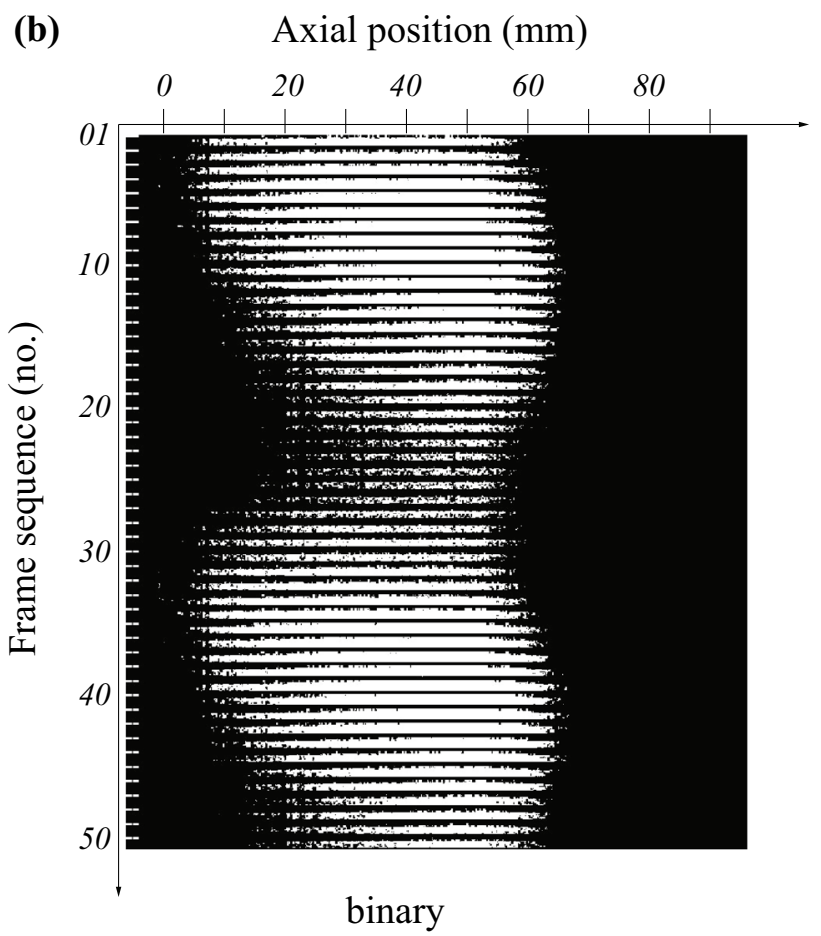

(b)
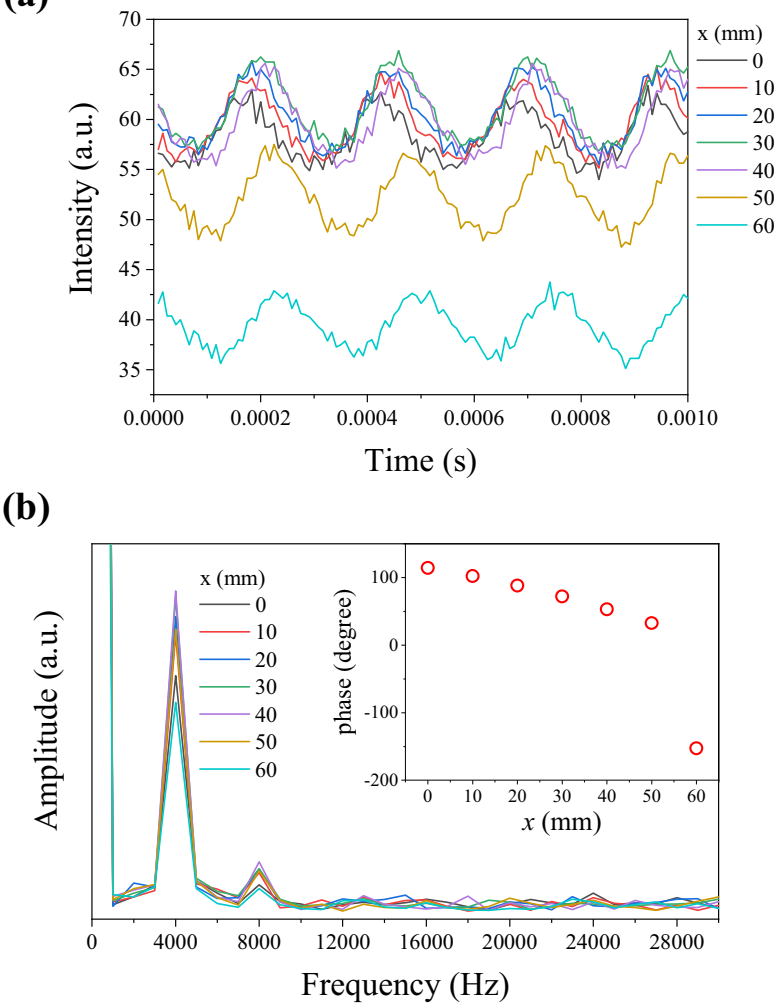

(c)

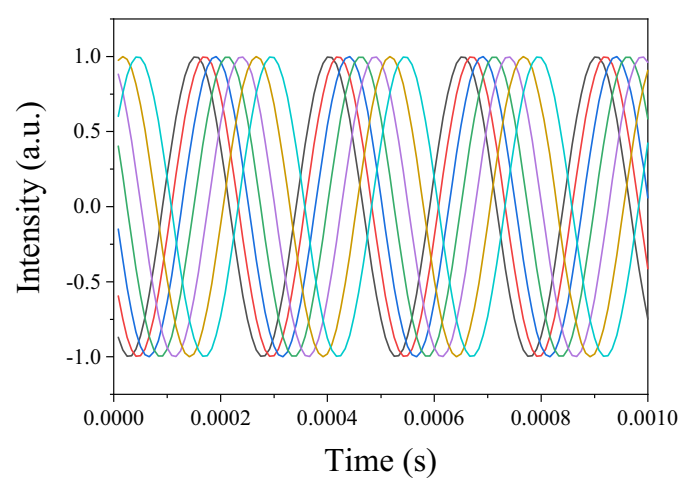

Fig. 6 The optical signal deduced from video captured by the highspeed video camera using ImageJ software. a Shows the optical emission intensity variations with time at seven different distances to the torch exit; b Shows the FFT results of the signals in the frequency domain with an inset showing the phases of each $4 \mathrm{kHz}$ signal at the seven positions; and $\mathbf{c}$ are the rebuilt specific signals using the phase values with normalized amplitudes

Fig. 5 Fifty frames of a continuous image sequence of the argon plasma plume. $\mathbf{a}$ Is in the original form and $\mathbf{b}$ is in the binary form

affect the result of the velocity calculation. For the sake of digital processing in FFT, $N$ should have the form $N=2^{n}$, where $n$ is a natural number.

\section{Experimental verification}

To verify the feasibility of SSIV, experiments have been conducted in a non-transferred direct current plasma torch where the plasma plume velocity was previously measured using a group of electrostatic probes. By setting Langmuir 


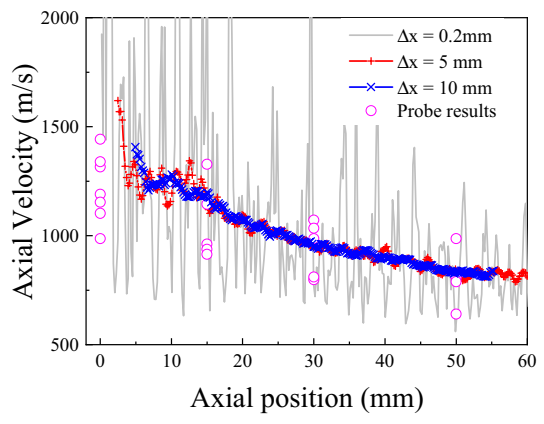

Fig. 7 Comparison of axial velocity distributions of an Ar plasma measured using SSIV and the Langmuir probe method

probes at given space points, the traveling time of an applied perturbation of the plasma plume was detected, and then the average axial velocity of the plasma flow was calculated.

In the present experiments, a laminar argon plasma plume was produced with an arc current of $100 \mathrm{~A}$, gas flow rate of $0.25 \mathrm{~g} / \mathrm{s}$ and chamber pressure of $120 \mathrm{~Pa}$. The arc voltage has an intrinsic fluctuation caused by a Helmholtz oscillation with a characteristic frequency of $\sim 4000 \mathrm{~Hz}$ (Huang et al. 2010), leading to corresponding energy fluctuations on the plasma (Huang et al. 2012). The relative fluctuation-amplitude is only several percentages, which is sufficient for SSIV diagnosis, and this frequency is distinctively far from the frequencies caused by other mechanisms. A high-speed video camera (MotionBLITZ ${ }^{\circledR}$ Cube3) with a bright $50 \mathrm{~mm}$ lens having a maximum aperture of 0.95 was used to record the optical intensity of the plasma plume. The camera lens was focused on the center of the plasma jet with a small depth of focus. The maximum frame rate of $1.2 \times 10^{5} \mathrm{fps}$ was used to facilitate higher temporal resolution at the expense of reduced CCD resolution to a relatively low value of $512 \times 10$ pixels. Experimental calibration shows that each pixel on the image corresponds to a spatial size of $0.188 \mathrm{~mm}$ on the focal plane. Therefore, a narrow strip with a size of $96 \times 1.9 \mathrm{~mm}$ near the axis of the plasma plume can be photographed in the present study to demonstrate the measurement of axial velocity distributions using SSIV. A computer code was developed to perform FFT analysis of the signals at each pixel based on the intensity spectrum obtained by ImageJ software. The phase shift was also calculated automatically with the code.

Figure 5a shows 50 continuous frames obtained using the high-speed video camera, which corresponds to a sampling time of $0.4 \mathrm{~ms}$. By setting intensity thresholds, Fig. 5a is transformed to binary form, as shown in Fig. 5b, which clearly indicates temporal and spatial plasma plume intensity variations. Not only does the intensity of the entire frame change with time, but the intensities of different positions also change. Because $f_{\mathrm{s}}$ is $\sim 4000 \mathrm{~Hz}, 1.6$ periods of variation occur in a sampling time of $0.4 \mathrm{~ms}$. A supplementary video metafile is attached in this paper, from which the variations following the flow are seen more clearly.

To quantitatively obtain the propagation process of the specific signal in the plasma plume, image analysis of the original images was conducted. Figure 6a shows the extracted results of the optical emission intensity fluctuations at seven axial positions from 0 to $60 \mathrm{~mm}$ with a separation of $10 \mathrm{~mm}$ in a sampling time of $1 \mathrm{~ms}$. It is apparent that the fluctuations of each curve have the same main frequency but different amplitudes and phases. Figure $6 \mathrm{~b}$ shows the FFT analysis results in the frequency domain. At different axial positions, the frequency spectrum is almost the same as the main peak at $4 \mathrm{kHz}$, indicating that the specific signal is well transmitted to various positions of the present laminar plasma flow. Because the selected sampling time is $1 \mathrm{~ms}$ and the sampling frequency equals $f_{\mathrm{a}}$, other low-frequency signals $<1 \mathrm{kHz}$ or high-frequency signals greater than $f_{\mathrm{a}}$ are not easily observable. In the inset of Fig. 6b, the initial phases of the signals at $4 \mathrm{kHz}$ are also shown, clearly giving the trend of the propagation of the signal. Although it is not necessary to rebuild the specific signal for each position to calculate the flow velocity, examples of the signal rebuilding are shown in Fig. $6 \mathrm{c}$ using the phase result in Fig. 6b, and the amplitudes are normalized, which agrees well with the detected signals in Fig. 6a.

Figure 7 shows the typical results for the axial velocity distribution with 320 data points. The measured results with a $\Delta x$ of $0.2 \mathrm{~mm}, 5 \mathrm{~mm}$ and $10 \mathrm{~mm}$ obtained via SSIV are compared with the velocity measured using an electric probe. When $\Delta x$ is $0.2 \mathrm{~mm}$, which is almost the same as the 1 pixel size of $0.188 \mathrm{~mm}$ mentioned previously, significant noise exists because the propagation time between two adjacent pixels is too small, which leads to large errors when it is used as a dividend in the velocity calculation. When $\Delta x$ is $5 \mathrm{~mm}$, the velocity obtained via SSIV agrees well with the result measured using the Langmuir probes. At the exit of the plasma torch, the axial velocity reaches $\sim 1500 \mathrm{~m} / \mathrm{s}$, and it decreases to $\sim 800 \mathrm{~m} / \mathrm{s}$ at the axial position of $60 \mathrm{~mm}$. The velocity oscillates near the torch exit. This is because, on one hand, noise might exist; on the other hand, the velocity of the plasma plume has reached the local speed of sound of the argon plasma for typical values of $930 \mathrm{~m} / \mathrm{s}$ and $1200 \mathrm{~m} / \mathrm{s}$ at 3000 and $5000 \mathrm{~K}$, respectively, where the effect of fluid compressibility becomes significant. When compression and expansion waves are present, the velocity of the flow no longer changes monotonically. The result with a $\Delta x$ of $10 \mathrm{~mm}$ is quite consistent with that of $5 \mathrm{~mm}$, while the oscillation is further evened. 


\section{Discussion}

\subsection{Applicability for a turbulent flow}

\subsubsection{Methodological amendment}

Although the situation becomes more complicated for plasma flow with turbulence, SSIV remains usable once some criteria are met. As stated by Taylor (1938), if the velocity of the air stream that carries the eddies is much greater than the turbulent velocity, one may assume that the sequence of changes in $u$ at the fixed point are simply due to the passage of an unchanging pattern of turbulent motion over the point. Therefore, as long as the specific signal is detectable, SSIV is applicable in turbulent flow. When the velocity is composed of the time averaged component $U_{0}\left(x_{i}\right)$ and the stochastic component $U_{1}\left(x_{i}, t\right)$, the phase evolving lines of the "stamp" will be superimposed with chirpings, which means that the observed specific frequency might fluctuate around $f_{\mathrm{s}}$. where $\left[U_{1}(x)\right]_{\mathrm{RMS}} / U_{0}(x)$ is a small quantity according to the first prerequisite. Hence, the observed specific signal remains recognizable because $f_{\mathrm{ob}}\left(x_{i}\right)$ is nearly equal to $f_{\mathrm{s}}$ and far from the other frequency component, ensuring the applicability of SSIV.

Regarding the methodology of the turbulent case, if the total signal processing time length $\Delta t=N / f_{\mathrm{a}}$ is smaller than the smallest time scale of turbulence, $\tau$, the flow can be treated as quasistatic, similar to the laminar case, and the measuring process as well. This criterion might be fulfilled if a superfast camera, such as a high-speed ICCD, is used. The smallest time scale, which represents the energy dissipation rate for small-scale motion, can be expressed by the Kolmogorov microscale of time (Tennekes and Lumley 1972) $\tau=\sqrt{v / \varepsilon}$.

If $\tau$ is smaller than $\Delta t$, the flow velocity shall be treated as a tempo variable. The capturing of the "stamp" and signal processing is the same as aforementioned, but the calculation process has a few amendments.

Similar to Eq. (3), the phase shift between two adjacent points is

$\Delta \phi_{i}=2 \pi f_{\mathrm{s}}\left(\int_{x_{i}}^{x_{i}+\Delta x} \frac{d x}{U_{0}(x)}+\int_{0}^{x_{i}+\Delta x} \frac{U_{1}(x, t)}{U_{0}(x)} \frac{d x}{U_{0}(x)}-\int_{0}^{x_{i}} \frac{U_{1}(x, t)}{U_{0}(x)} \frac{d x}{U_{0}(x)}\right) \approx 2 \pi f_{\mathrm{s}} \frac{\Delta x}{\bar{U}_{0}\left(x_{i}\right)}\left(1+\frac{\bar{U}_{1}\left(x_{i}, t\right)}{\bar{U}_{0}\left(x_{i}\right)}\right)$,

However, it is possible to apply SSIV under several prerequisites for turbulence.

(i) $\left|U_{1}\left(x_{i}, t\right)\right|<<U_{0}\left(x_{i}\right)$;

(ii) (ii) the turbulence is statistically steady.

Under these conditions, the chirpings might be much smaller, and Eq. (2) should be rewritten with the first-order approximation as where the upper bar is averaged in $x_{i} \sim x_{i}+\Delta x$, and the second order small quantity is omitted. The calculated velocity from the phase shift $\Delta \phi_{i}$ and specific frequency $f_{\mathrm{s}}$ is

$U_{\text {cal }}\left(x_{i}, t\right)=\frac{2 \pi f_{\mathrm{s}} \Delta x}{\Delta \phi_{i}} \approx \bar{U}_{0}\left(x_{i}\right)-\bar{U}_{1}\left(x_{i}, t\right)$

which embodies the stochastic component of the turbulent velocity. If combined with a repeating measuring procedure,

$I\left(x_{i}, t\right)=A\left(x_{i}\right) \sin \left[2 \pi f_{\mathrm{s}}\left(t-\int_{0}^{x_{i}} \frac{d x}{U_{0}(x)+U_{1}(x, t)}\right)\right]=A\left(x_{i}\right) \sin \left[2 \pi f_{\mathrm{s}}\left(t+\int_{0}^{x_{i}} \frac{U_{1}(x, t)}{U_{0}(x)} \frac{d x}{U_{0}(x)}-\int_{0}^{x_{i}} \frac{d x}{U_{0}(x)}\right)\right]$.

The observed specific frequency can be deduced by partially differentiating the phase with time,

$f_{\mathrm{ob}}\left(x_{i}\right)=f_{\mathrm{s}}\left(1+\int_{0}^{x_{i}} \frac{1}{U_{0}(x)} \frac{\partial U_{1}(x, t)}{\partial t} \frac{d x}{U_{0}(x)}\right)$.

Then,

$$
\begin{gathered}
\left|f_{\mathrm{ob}}\left(x_{i}\right)-f_{\mathrm{s}}\right|<f_{\mathrm{s}} \int_{0}^{x_{i}} \frac{1}{U_{0}(x)}\left|\frac{\partial U_{1}(x, t)}{\partial t}\right| \frac{d x}{U_{0}(x)} \\
\approx f_{\mathrm{s}} \frac{1}{\tau} \int_{0}^{x_{i}} \frac{\left[U_{1}(x)\right]_{\mathrm{RMS}}}{U_{0}(x)} \frac{d x}{U_{0}(x)}<<f_{\mathrm{s}},
\end{gathered}
$$

the statistic velocity and turbulent velocity can be determined by averaging:

$$
\left\{\begin{array}{l}
\bar{U}_{0}\left(x_{i}\right)=\overline{U_{\text {cal }}\left(x_{i}, t\right)} \\
\bar{U}\left(x_{i}, t\right)=2 \overline{U_{\text {cal }}\left(x_{i}, t\right)}-U_{\text {cal }}\left(x_{i}, t\right)
\end{array}\right.
$$


(a) Axial position ( $\mathrm{mm})$

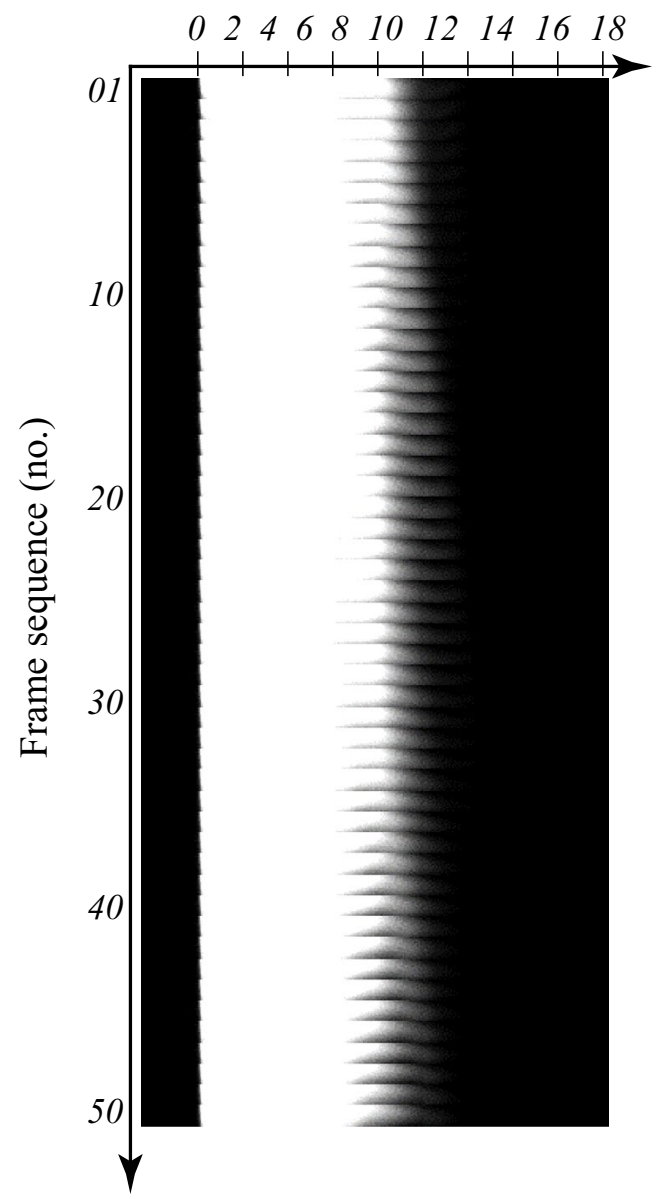

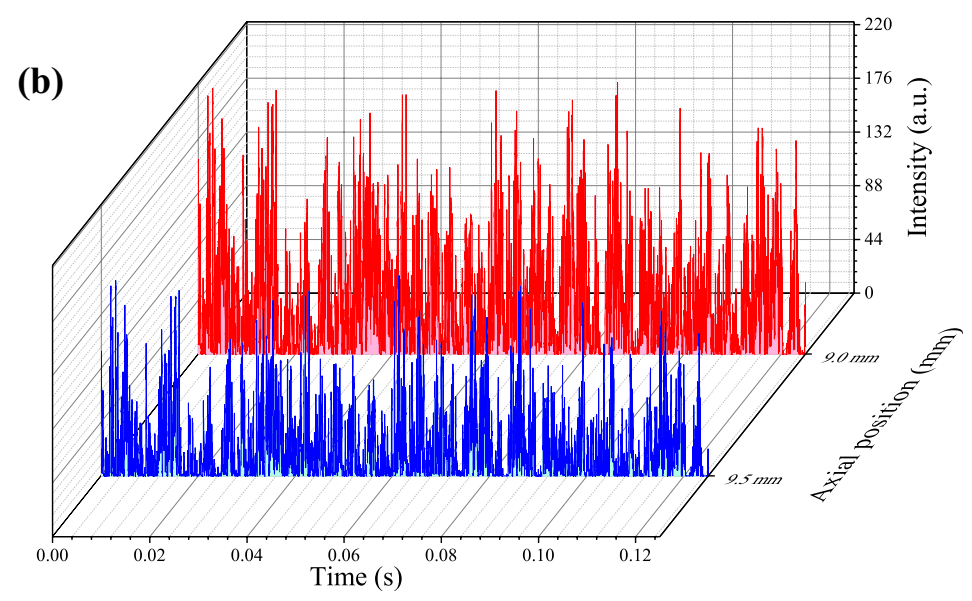

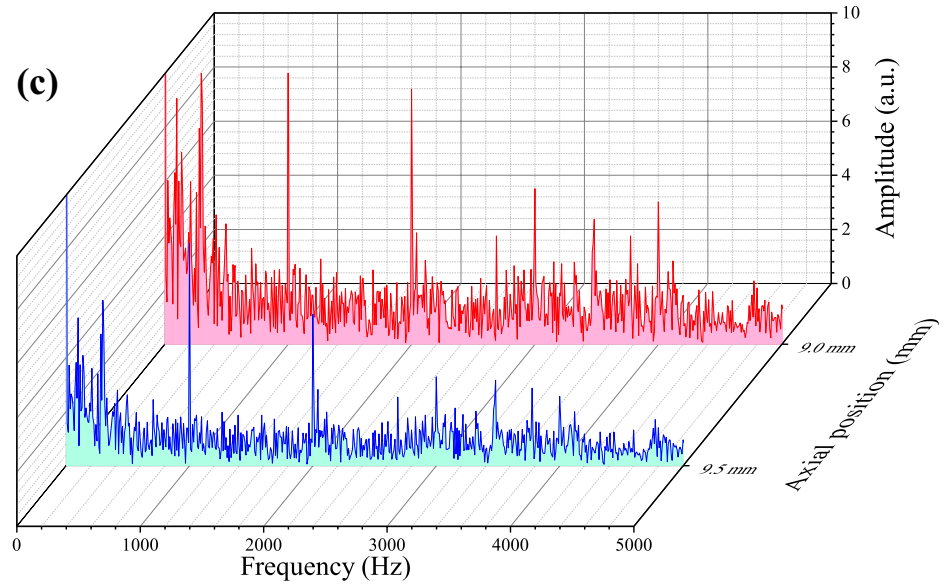

Fig. 8 Experimental results of a turbulent flow. a Fifty frames of a continuous image sequence of the turbulent plasma jet; $\mathbf{b}$ intensity variations at $x=9.0$ and $x=9.5 \mathrm{~mm}$ in $0.125 \mathrm{~s}$ and (c) the corresponding frequency spectra

\subsubsection{Experimental verification}

To verify the applicability in turbulent flow, experiments are conducted in a turbulent plasma torch working in atmosphere. The working gas is argon with $20 \mathrm{slm}$, and the input power is $\sim 2 \mathrm{~kW}$. An external bypass circuit with a resistance tenfold the arc resistance is connected in parallel with the arc torch. The connection of the bypass circuit is switched at $1 \mathrm{kHz}$ with a duty ratio of 0.5 to produce a specific signal externally. Therefore, the influence of the bypass circuit on the arc reduces the arc power by $<5 \%$. The frequency is chosen to be sufficiently small to prevent the alternating signal from being averaged in the torch and should be sufficiently large to contain several periods in the image sequence. Under the experimental conditions, the plasma jet is in a typical turbulent state.

A faster video camera (IX Camera, $i$-speed 513) is used to capture the optical emission with a frame rate of
$5.0 \times 10^{5} \mathrm{fps}$. The image resolution is reduced to $392 \times 18$ pixels and located near the center of the jet with a size of $20.60 \times 0.95 \mathrm{~mm}$. Figure 8 a shows 50 continuous frames obtained by the high-speed video camera, which corresponds to a sampling time of $1.0 \mu \mathrm{s}$. The jet clearly fluctuates at the tail, while at the core region, the image is oversaturated. To demonstrate the feasibility of SSIV in such a flow, two positions with $x=9.0 \mathrm{~mm}$ and $9.5 \mathrm{~mm}$ are chosen for axial velocity evaluation.

The optical intensity variations and the frequency spectra are extracted from an image sequence of 62,500 frames, corresponding to a time interval of $0.125 \mathrm{~s}$. The results are shown in Fig. 8b, c. The optical intensity varies significantly with time, revealing the turbulent nature of the flow. Although the intensity is in an arbitrary unit, the mean intensity of 39.67 at $9 \mathrm{~mm}$ is much larger than that of 20.19 at $9.5 \mathrm{~mm}$, indicating a substantial temperature decrease of the plasma. Comparing Figs. 8b, 6a shows that it is almost impossible to evaluate $\Delta t$ in turbulent flow 
using the intensity comparison method. However, as stated before, the phase shift of the specific signal can help. The applied specific signal of $1 \mathrm{kHz}$ and its harmonies are easily distinguishable in the frequency spectra, and the phase shift between the two positions is approximately $0.79^{\circ}$, leading to a calculated velocity of $241 \mathrm{~m} / \mathrm{s}$ using Eq. (9), which is quite reasonable in the present experimental conditions.

\subsection{Discussion on the applied specific signal and its distortion}

The externally applied specific signal is proposed as the "stamp" for SSIV if there is no proper specific signal in the flow. The externally applied signal, as mentioned, should not arouse substantial changes in the flow velocity, especially when it is applied in the input power. In Sect. 4.1.2, the perturbed input power is $<5 \%$, and then the relative change in velocity is $<2.5 \%$ since

$\frac{P}{\dot{m}}=\frac{U^{2}(x)}{2}+c_{p} T>\frac{U^{2}(x)}{2} \Rightarrow \frac{\Delta U(x)}{U(x)}<\frac{1}{2} \frac{\Delta P}{P}$.

The tempo variable velocity perturbation might cause a distortion of the observed frequency of the specific signal. To quantitatively discuss the distortion, assuming that the applied specific signal arouses a small velocity oscillation with the same frequency, the velocity has the form:

$U(x)\left(1+\varepsilon \cdot \sin 2 \pi f_{\mathrm{s}} t\right)$,

where $\varepsilon$ is the ratio of the velocity perturbation to the unperturbed velocity. Then, Eq. (2) changes to

$I\left(x_{i}, t\right)=A\left(x_{i}\right) \sin \left[2 \pi f_{\mathrm{s}}\left(t-\frac{1}{1+\varepsilon \cdot \sin 2 \pi f_{\mathrm{s}} t} \int_{0}^{x_{i}} \frac{d x}{U(x)}\right)\right]$.

Then, the observed specific frequency is

$f_{\mathrm{ob}}\left(x_{i}\right)=f_{\mathrm{s}}\left(1-\frac{\varepsilon \cdot \cos 2 \pi f_{\mathrm{s}} t}{\left(1+\varepsilon \cdot \sin 2 \pi f_{\mathrm{s}} t\right)^{2}} \int_{0}^{x_{i}} \frac{2 \pi f_{\mathrm{s}} d x}{U(x)}\right)$.

If $\varepsilon<<1$, then,

$f_{\mathrm{ob}}\left(x_{i}\right) \approx f_{\mathrm{s}}\left(1-\varepsilon \cdot \int_{0}^{x_{i}} \frac{2 \pi f_{\mathrm{s}} d x}{U(x)} \cdot \cos 2 \pi f_{\mathrm{s}} t\right)$.

This demonstrates that the temporal perturbation of the velocity couples with the drift of the fluid, causing a modulation of the observed frequency. The modulus of the frequency modulation is a linear function of the relative perturbation of the velocity and the drifting time from the position where the specific signal is applied to the measuring position. In the previous Sect. 4.1.2, the modulus of the frequency modulation is approximately

$\varepsilon \cdot \int_{0}^{x_{i}} \frac{2 \pi f_{\mathrm{s}} d x}{U(x)} \leq 0.025 \times \frac{2 \pi \times 1000 \mathrm{~Hz} \times 9.0 \mathrm{~mm}}{241.41 \mathrm{~m} / \mathrm{s}}=5.8 \times 10^{-3}$.

Such a small-scale frequency shift, as previously illustrated in Fig. 4, does not affect the SSIV process and can be omitted.

\subsection{Other possible specific signal sources and detection methods}

Electromagnetic signals are not the only candidate source for SSIV; other signals can play the same role once they form distinguishable patterns either in spatial or temporal dimensions, and the most basic characteristic, e.g., the frequency, is not substantially interfered with by other sources in the process under consideration. In other words, if a signal has at least one certain characteristic which has only translation or shift without substantial changes in the amplitude, the signal might be used for SSIV. As shown in the previous examples, instead of the intensity, it is the frequency that is used to distinguish the specific signal, with the phase shift being used to evaluate the velocity. One possible example is using a high repetition frequency laser-induced fluorescence spectrum as the source.

At the same time, imaging is not the only detection method. To capture the pattern or the "stamp", a conventional diagnostic method can be applied, including spectroscopy for line emissions and Schlieren visualization for density gradients.

\section{Conclusion}

Combining the image process technique and signal process method, SSIV is proposed as a novel velocity-measurement method using a series of small volumes with different states of the flow in the time domain as the tracer. SSIV can be applied in rarefied flow with high velocity. The algorithm and measurement feasibility are illustrated, and their possibility of being applied in turbulent flow is elucidated. SSIV is experimentally verified, and the results agree well with electrical probe measurements, which indicates that SSIV is a promising technique for quick and easy flow velocity distribution measurements.

Acknowledgements This work was supported by the Strategic Priority Research Program of the Chinese Academy of Sciences (Grant No. XDA17030100) and the National Natural Science Foundations of China under Grant 11735004 and Grant 11575273. 


\section{References}

Acrivlellis M (1980) Measurements by means of triple-sensor probes (turbulent flows). J Phys E Sci Instrum 13:986

Ahedo E (2011) Plasmas for space propulsion. Plasma Phys Contr Fusion 53:124037. https://doi.org/10.1088/07413335/53/12/124037

Auweter-Kurtz M, Glocker B, Golz T, Kurtz HL, Messerschmid EW, Riehle M, Zube DM (1996) Arcjet thruster development. J Propul Power 12:1077-1083. https://doi.org/10.2514/3.24146

Boulos MI, Fauchais P, Wender E (1997) Thermal plasmas: Fundamentals and applications volume 1 vol 14. vol 2. Plenum Press, New York

Brossa M, Pfender E (1988) Probe measurements in thermal plasma jets. Plasma Chem Plasma Process 8:75-90. https://doi. org/10.1007/bf01016932

Bufton SA, Burton RL (1997) Velocity and temperature measurements in a low-power hydrazine arcjet. J Propuls Power 13:768-774. https://doi.org/10.2514/2.5231

Chapman S, Cowling TG (1958) The mathematical theory of nonuniform gases.

Chazot O, Panerai F (2015) High-enthalpy facilities and plasma wind tunnels for aerothermodynamics ground testing.

Coudert JF, Planch MP, Fauchais P (1995) Velocity measurement of dc plasma jets based on arc root fluctuations. Plasma Chem Plasma Process 15:47-70. https://doi.org/10.1007/bf01596681

Dubreus T, Sheeley J, Stewart J Development of a mid-pressure archeated facility for hypersonic vehicle testing. In: Us Air Force T\&e Days, 2013.

Farmer WM, Brayton DB (1971) Analysis of atmospheric laser Doppler velocimeters. Appl Opt 10:2319-2324. https://doi. org/10.1364/AO.10.002319

Fauchais P (2004) Understanding plasma spraying. J Phys D Appl Phys 37:R86-R108. https://doi.org/10.1088/0022-3727/37/9/r02

Fauchais P, Vardelle A (1997) Thermal plasmas. IEEE Trans Plasma Sci 25:1258-1280. https://doi.org/10.1109/27.650901

Fauchais P, Coudert JF, Vardelle M (1989) 7 - Diagnostics in thermal plasma processing. In: Auciello O, Flamm DL (eds) Plasma Diagnostics. Academic Press, pp 349-446. doi:https://doi.org/https:// doi.org/10.1016/B978-0-12-067635-4.50012-0

Fauchais PL, Heberlein JVR, Boulos MI (2014) Overview of thermal spray. In: Thermal Spray Fundamentals: From Powder to Part. Springer US, Boston, MA, pp 17-72. https://doi.org/10.1007/9780-387-68991-3_2

Gendrich CP, Koochesfahani MM (1996) A spatial correlation technique for estimating velocity fields using molecular tagging velocimetry (MTV). Exp Fluids 22:67-77

Gendrich CP, Koochesfahani MM, Nocera DG (1997) Molecular tagging velocimetry and other novel applications of a new phosphorescent supramolecule. Exp Fluids 23:361-372

Huang H, Eguchi K, Yoshida T (2003) Novel structured yttria-stabilized zirconia coatings fabricated by hybrid thermal plasma spraying. Sci Technol Adv Mater 4:617-622. https://doi.org/10.1016/j. stam.2004.01.004

Huang H, Pan WX, Guo ZY, Wu CK (2010) Instabilities in a non-transferred direct current plasma torch operated at reduced pressure. J Phys D Appl Phys 43:085202. https://doi.org/10.1088/00223727/43/8/085202

Huang H, Pan W, Wu C (2012) Energy fluctuations in a direct current plasma torch with inter-electrode inserts operated at reduced pressure. Plasma Chemistry Plasma Process 32:65-74. https://doi. org/10.1007/s11090-011-9331-2

Johnson LK, Bromaghim DR, Dulligan MW, Hoskins WA, Spanjers GG (2015) On-orbit optical observations of electric propulsion space experiment 26 kilowatt Arcjet. J Propuls Power 18:763-767
Johnson BH, Murphree DL (1969) Plasma velocity determination by electrostatic probes. AIAA J 7:2028-2030. https://doi. org/10.2514/3.5504

Kado S, Shikama T, Kajita S, Oishi T, Tanaka S (2004) Plasma flow measurements in linear divertor simulator MAP-II using Mach probe and directional Langmuir probe. Contribu Plasma Phys 44:656-661

Kambara M et al (2014) Nano-composite Si particle formation by plasma spraying for negative electrode of $\mathrm{Li}$ ion batteries. J Appl Phys 115:143302. https://doi.org/10.1063/1.4870600

Kawall JG, Shokr M, Keffer JF (2006) A digital technique for the simultaneous measurement of streamwise and lateral velocities in turbulent flows. J Fluid Mech 133:83-112

Keane RD, Adrian RJ (1990) Optimization of particle image velocimeters: II. Multiple pulsed systems. Meas Sci Technol 1:1202-1215

Kohli R (2012) Chapter 5 - Developments in imaging and analysis techniques for micro- and nanosize particles and surface features. In: Kohli R, Mittal KL (eds) Developments in Surface Contamination and Cleaning. William Andrew Publishing, Oxford, pp 215-306. https://doi.org/10.1016/B978-1-4377-7883-0.00005-5

Lekakis IC, Adrian RJ, Jones BG (1989) Measurement of velocity vectors with orthogonal and non-orthogonal triple-sensor probes. Exp Fluids 7:228-240. https://doi.org/10.1007/BF00198002

Lempert WR, Jiang N, Sethuram S, Mo S (2002) Molecular tagging velocimetry measurements in supersonic microjets. AIAA J 40:1065-1070

Lyn DA, Einav S, Rodi W, Park JH (1995) A laser-Doppler velocimetry study of ensemble-averaged characteristics of the turbulent near wake of a square cylinder. J Fluid Mech 304:285-319. https://doi. org/10.1017/S0022112095004435

Maclatchy CS, Boucher C, Poirier DA, Gunn J (1992) Gundestrup: a Langmuir/Mach probe array for measuring flows in the scrape-off layer of TdeV. Rev Sci Instrum 63:3923

Mazouffre S (2016) Electric propulsion for satellites and spacecraft: established technologies and novel approaches. Plasma Sour Sci Technol 25:033002. https://doi.org/10.1088/09630252/25/3/033002

Metcalfe J, Quigley M (1975) Heat transfer in plasma-arc welding. Weld J 54:99-103

Optical Flow and Trajectory Estimation Methods (2016). SpringerBriefs in Computer Science.

Pan W, Meng X, Huang H, Wu C (2016) Producing ultra-high-speed nitrogen jets by arc heating in a low-pressure chamber. Theor Appl Mech Lett 6:60-63

Peterson BJ, Talmadge JN, Anderson DT, Anderson FSB, Shohet JL (1994) Measurement of ion flows using an "unmagnetized", Mach probe in the interchangeable module stellarator. Rev Sci Instrum 65:2599-2606

Pfender E (1999) Thermal plasma technology: where do we stand and where are we going? Plasma Chem Plasma Process 19:1-31

Planche MP, Coudert JF, Fauchais P (1998) Velocity measurements for arc jets produced by a DC plasma spray torch. Plasma Chem Plasma Process 18:263-283. https://doi.org/10.1023/a:10216 06701022

Raffel M, Willert CE, Scarano F, Kähler CJ, Wereley ST, Kompenhans J (2017) Particle image velocimetry—a practical guide, 3rd edn. Springer, Switzerland

Ramakrishnan S, Gershenzon M, Polivka F, Kearney TN, Rogozinski MW (1997) Plasma generation for the plasma cutting process. IEEE Trans Plasma Sci 25:937-946. https://doi. org/10.1109/27.649600

Segall J, Brown MS, Skaggs PA, Debarber PA, Pitz RW, Nandula SP, Brown TM (1996) Unseeded velocity measurement by ozone tagging velocimetry. Opt Lett 21:755-757 
Shen Q (2005) Rarefied gas dynamics—fundamentals, simulations and micro flows, vol 3. Springer, Germany

Taylor GI (1938) The spectrum of turbulence. Proc R Soc Lon Ser-A 164:0476-0490

Tennekes H, Lumley JL (1972) A first course in turbulence. The MIT Press, Cambridge

Underwood TC, Loebner KTK, Miller VA, Cappelli MA (2020) Schlieren diagnostic for cinematic visualization of dense plasma jets at Alfvenic timescales. Exp Fluids 61:17

V Le, M B, D G (2003) Thermal spray 2003: advancing the science and applying the technology. In: Paper presented at the ASM International Materials Park. Vol. 2, Ohio, USA

Wang Q, Wu Y, Cheng HT, Mei XH, Zhao CY (2019) A schlieren motion estimation method for seedless velocimetry measurement. Exp Therm Fluid Sci 109:109880. https://doi.org/10.1016/j.expth ermflusci.2019.109880
Wehrmeyer JA, Ribarov LA, Oguss DA, Pitz RW (1999) Flame flow tagging velocimetry with $193-\mathrm{nm}_{2} \mathrm{O}$ photodissociation. Appl Opt 38:6912

Westerweel J, Elsinga GE, Adrian RJ (2013) Particle image velocimetry for complex and turbulent flows. Annu Rev Fluid Mech 45:409

Wilson F (2006) Recent advances in satellite propulsion and associated mission benefits. In: Aiaa International Communications Satellite Systems Conference, 2006

Zhang S, Yu X, Yan H, Huang H, Liu H (2017) Molecular tagging velocimetry of $\mathrm{NH}$ fluorescence in a high-enthalpy rarefied gas flow. Appl Phys B 123:122. https://doi.org/10.1007/s0034 0-017-6703-1

Zhukov MF, Zasypkin IM (2007) Thermal plasma torches: design, characteristics, application. Cambridge International Science Publishing, Cambridge

\title{
Affiliations
}

\author{
Jinwen Cao ${ }^{1} \cdot$ Heji Huang ${ }^{1,2}$ (]) Wenxia Pan ${ }^{1,2} \cdot$ Xian Meng ${ }^{1} \cdot$ Chengkang Wu $\mathbf{u}^{1,2}$ \\ Heji Huang \\ huang@imech.ac.cn \\ 2 School of Engineering Science, University \\ of Chinese Academy of Sciences, Beijing 100049, \\ People's Republic of China
}

1 State Key Laboratory of High Temperature Gas Dynamics, Institute of Mechanics, Chinese Academy of Sciences, Beijing 100190, People's Republic of China 